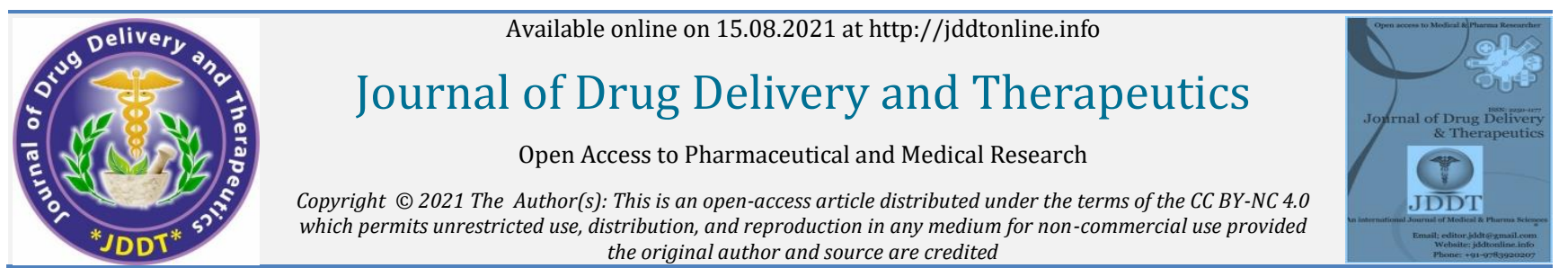

Open Access Full Text Article the original author and source are credited
antion

Review Article

\title{
Transdermal patches: an emerging mode of drug delivery system in pulmonary arterial hypertension
}

\author{
(DDubey Rupal* and (DPothuvan Umadoss \\ School of Pharmacy and Research, People's University, Bhanpur, 462037 Bhopal, M.P India
}

\section{Article Info:}

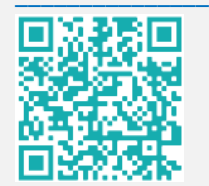

\section{Article History:}

Received 07 June 2021

Reviewed 13 July 2021

Accepted 22 July 2021

Published 15 August 2021

\section{Cite this article as:}

Dubey R, Pothuvan U, Transdermal patches: an emerging mode of drug delivery system in pulmonary arterial hypertension, Journal of Drug Delivery and Therapeutics. 2021; 11(4-S):176-186

DOI: http://dx.doi.org/10.22270/jddt.v11i4-S.4925

\section{*Address for Correspondence:}

Dr. Rupal Dubey, School of Pharmacy and Research, People's University, Bhopal (M.P)

ORCID ID: https://orcid.org/0000-0002-7033-8782

\begin{abstract}
Transdermal Patches have been contributing important part to the pharmaceutical industry and medical practice by providing advances in delivery of treatment with existing and nove drugs. Transdermal drug delivery system has made great contribution in the medical practices but many researches are undergoing to achieve its full potential. Transdermal drug delivery system was came into existence to overcome difficulties of drug delivery especially oral route. Transdermal drug delivery refers to means of delivering drugs through the surface of the skin for local or systemic treatment. The drug functions after absorption through skin into the systemic circulation via capillary action at certain rate. Transdermal patches are now widely used as topical and transdermal delivery systems. These patches are a significant result of advancements in skin science, technology, and knowledge, which have been created via trial and error, clinical observation, and evidence-based investigations dating back to the earliest human records. A transdermal patch is a medicated adhesive patch that is applied to the skin and used to deliver a precise amount of medicine into the bloodstream via the skin. A benefit of transdermal medication administration over other forms of delivery systems such as oral, topical, intravenous (i.v.), intramuscular (i.m.), and so on is that it is non-invasive. Transdermal patches provide medication to the patient in a regulated manner, either by a porous membrane covering a reservoir of medication or by body heat melting tiny layers of drug contained in the adhesive. This review article covers the basics of transdermal patches, such as the many types of patches, how they're made, and what factors influence them, among other things.
\end{abstract}

Keyword: Skin Delivery, Transdermal Drug Delivery System, Transdermal Excipients, Pulmonary Arterial Hypertension, Sildenafil Citrate.

\section{INTRODUCTION}

First transdermal patch approved in 1979 by FDA was of Scopolamine for motion sickness. Nitroglycerine was the second patch authorized in 1981. A large variety of patches for transdermal application are available on the market. Few of those are: Testosterone, Nicotine, Fentanyl, Clonidine, and Hormones etc. those patches usually applied minimum 1 day to maximum 7 days $^{1}$ depending upon therapeutic requirement. Transdermal delivery not only allows for continuous, predetermined, and consistent drug administration, but also allows for regulated input of medicines with short biological half-lives and prevents pulsed entrance into systemic circulation, avoiding unwanted side effects. The oral route is the most common method of drug delivery, but it has some drawbacks, such as first pass metabolism (the rapid uptake and metabolism of an agent by the liver into inactive compounds immediately after enteric absorption and before it reaches the systemic circulation), drug degradation in the gastrointestinal tract due to enzymes, $\mathrm{pH}$, and other factors. A new medication delivery method based on transdermal patches was developed to address these issues ${ }^{2}$. A Transdermal Drug Delivery System (TDDS) is a method of delivering drugs via the skin for local or systemic therapeutic effects. It is, together with oral medicine and injectable, one of the primary study topics for third-generation pharmacological preparations ${ }^{3}$. The drug's delivery technique, which is easy, simple to use, non-invasive, and improves patient compliance, is one of the reasons behind this ${ }^{4}$. TDDS also minimizes drug concentration fluctuations in the blood, maintains stable plasma levels, reduces the risk of overdosing, and facilitates drug detection ${ }^{5,6}$. Simultaneously, it avoids issues associated with oral administration, such as the impact of the gastrointestinal environment $(\mathrm{pH}$, enzyme activity, drug-food interaction) on therapeutic effectiveness and the 'first pass effect.' Transdermal drug delivery systems were commonly used to treat various skin disorders. Also, substantive applications have been found in the management of angina pectoris, pains, smoking, cessation \& neurological disorders such as Parkinson's disease ${ }^{7,8}$. Transdermal patches, are one of the novel pharmaceutical dosage form for the delivery of drugs upon application to the skin and into the bloodstream. By nature the patches are expected to provide controlled/sustained/modified delivery of drugs for defined period of time with predefined rate. Because the skin is such an efficient barrier, only medicines with a low molecular weight may be administered this way. Transdermal patches are currently accessible in a wide range of medicines. The first commercially available 
prescription patch was authorized by the US Food and Drug Administration in December 1979. Since then transdermal drugs continued to gain popularity along with further improvements to improve safety and efficacy. Further major step was the production of patches delivering peptide and even protein substances including growth hormone, insulin, and vaccines ect ${ }^{9}$. Transdermal patches can be categorized into three categories - first generation, second generation, and third generation. They are available in different sizes \& having more than one ingredients. Once they apply on normal skin they deliver active ingredients into systemic circulation passing through skin barrier. A transdermal patch containing high dose of drug inside is retained on the skin for prolonged period of time and enters into blood systemic circulation by diffusion process.

The evolution of transdermal delivery methods may be divided into three generations. Many presently used medicines that may penetrate the skin at predetermined therapeutic rates are classified as first generation. Second generation representing additional advancement in the system for small molecule delivery via skin and the Third generation allowing transdermal delivery of macromolecules (including proteins and DNA) and virusbased/other vaccines through targeted permeabilization of the skin's stratum corneum. In this review, we describe the transdermal delivery methods in each generation along with pros and cons thereof.

\section{Skin and its composition}

The most visible and largest organ of the body is skin. The skin covers a total surface area of approximately 1.5-2 $\mathrm{m}^{2}$ and is the barrier between human body and the external environment ${ }^{10}$. Temperature regulation, immunity against pathogens, electrolyte balance, and protection from physical traumas, chemical agents, and UV radiation are only a few of its tasks ${ }^{11,12}$. In addition, skin is also an important avenue for absorption of drugs and exerting their effect. The epidermis, dermis, and subcutaneous tissue of the skin are made up of appendages such as hair follicles, sebaceous glands, sweat glands, blood vessels, lymphatic vessels, nerves, and so on. The epidermis can be divided into five layers from the inside to the outside, namely the stratum basale, stratum spinosum, stratum granulosum, stratum lucidum, and stratum corneum (SC) ${ }^{12,13}$. The properties of SC are completely different from those of the other layers, with the entire structural construction by inactive keratinocytes and intercellular lipids that form a 'brick and mortar' model, where, the protein-rich keratinocytes serve as the bricks and the intercellular lipids serve as the mortar ${ }^{14,15}$. SC is the main factor determining the skin barrier, and also the major obstacle limiting the rate of percutaneous absorption even though the thickness is only $10-20 \mu \mathrm{m}^{16,17}$. Permeation of drug to the skin happens through two routes. The first is through skin appendages' inherent channels. These channels are hydrophilic and only a few microns in diameter. Owning to the fact that the average follicular orifice area on the human skin surface is only about $0.1 \%$ of the total surface area 18 , it is not the primary pathway of percutaneous absorption. The second pathway involves penetrating the epidermis, entering the dermis via the SC and deeper epidermis, and then being absorbed into the bodily circulation via capillaries. As for the penetration of drugs and passing through the SC, two pathways exist, namely, the transcellular route, through which substances infiltrate the keratinocytes and intercellular lipids, and subsequently pass through and are transported. Because the drug must diffuse through both hydrophilic and hydrophobic regions, it may not be suitable for most medicines. The intercellular route is the second and most likely route of drug delivery when it comes to entering the SC. It involves a convoluted journey via the lipids surrounding the keratinocytes ${ }^{19}$.

\section{First generation transdermal patches}

They are the first type of patches and have been used much in clinics. The transdermal patch design consists of a drug in a reservoir that is enclosed on one side with impermeable backing and an adhesive, which contacts the skin ${ }^{9}$. However, not all drugs with suitable properties can be delivered, due to certain limitations. The first generation transdermal patches are limited primarily to the outermost skin barrier that is stratum corneum. The stratum corneum is made up of dead cells (corneocytes), and it is the epidermis' outermost layer. This layer is made up of 15-20 layers of flattened cells that lack nuclei and cell organelles. Filamentous keratin may be seen in their cytoplasm. Corneocytes are encased in a lipid matrix that includes fatty acids, cholesterol, and ceramides. Hence, the drugs should be of low molecular weight, lipophilic, and efficient at low doses. The mechanism of transport through inter-cellular spaces allows diffusion of non-polar solutes or lipophilic solutes through the continuous lipid matrix. The transappendegeal route involves the passage of molecules through skin surface sweat glands and across the hair follicles.

\section{Second generation transdermal patches}

The second generation transdermal patches consists of advanced system that allows increased the skin permeability, reduced damage to the deeper tissues and better transport into the skin. However, enhancement methods developed in this generation, such as chemical enhancers, non-cavitation ultrasound and iontophoresis experienced difficulty in balancing between increased permeation through stratum corneum and at the same time protection of tissues at the deeper level. Chemical enhancers disrupt the highly ordered bilayer of the stratum corneum by inserting amphiphilic molecules to help in better permeation. This leads to skin irritation. Similarly, Iontophoresis involves administration of drugs into the stratum corneum under low voltage current. It primarily acts as an electrical motor for transmission over the stratum corneum. It does not disturb the skin barrier, thus, can be used for delivery of small molecules that carry a charge and some macromolecules up to a few Daltons. Rate of drug delivery can be controlled using a microprocessor. In Noncavitation ultrasound, the pressure gradients and oscillation associated with ultrasound emerges to be responsible for passage of drug through the skin by disrupting stratum corneum and thereby increasing the permeability. Ultrasound's effects have thus far been restricted to tiny lipophilic compounds. It's been restricted because of the tissue warmth it causes, which can harm deeper tissue ${ }^{20}$.

\section{Third generation transdermal patches}

The third generation of transdermal delivery systems uses a tailored strategy to disturb the stratum corneum while maintaining the integrity of deeper tissues. In human clinical trials, sophisticated methods such as novel chemical enhancers, thermal ablation, cavitational ultrasound, electroporation, microneedles, and microdermabrasion are used to transport macromolecules such as therapeutic proteins, vaccinations, and growth hormones across the $\operatorname{skin}^{21}$.

\section{ADVANTAGES OF TRANSDERMAL PATCHES 22}

An advantage of a transdermal drug delivery route over other types of medication delivery such as intravenous (i.v), intramuscular (i.m), oral, topical etc. The advantages of the transdermal patches are mentioned below. 
$\checkmark$ Bypassing the initial hepatic metabolism and infusing the medication continuously for longer periods of time without interference by GIT fluids, the drug's systemic bioavailability is increased.

$\checkmark$ The simplified medication regimen leads to improved patient compliance and comfort via non-invasive, painless and simple application.

$\checkmark$ Steady permeation of drug across the skin, allowing consistent plasma levels, but non-invasive in nature.

$\checkmark$ Drug therapy may be stopped immediately by removal of its application from the surface of the skin.

$\checkmark$ Shorter biological half-life drug can be permitted to administer slowly.

$\checkmark$ Drugs that cause gastrointestinal upset can be a good candidate for transdermal delivery because this method.

$\checkmark \quad$ It is not dependent on inter-individual \& intra-individual variation and hence increases therapeutic efficacy.

$\checkmark \quad$ In case of any adverse effect of poisoning transdermal patches can easily be removed from the surface of skin.

\section{DISADVANTAGES OF TRANSDERMAL PATCHES22}

The major disadvantage of transdermal delivery methods is that skin is a highly efficient barrier; as a result, only medicines with tiny enough molecules to permeate the skin may be administered this way. Also other disadvantages of the transdermal patches are mentioned below.

$\checkmark$ Transdermal application may lead to allergic reactions to the skin due to drug, adhesive or other excipients.

$\checkmark$ The use of transdermal delivery may be more expenditure.

$\checkmark$ Skin's impermeability creates hindrance against drug entry, so only potent API can be administered via this route.

$\checkmark$ Drug which has large molecular size gets difficulty in absorption.

$\checkmark$ Drugs which is ionic in nature gets problems in absorption.

$\checkmark$ Drugs which has high or very low partition coefficient fails to enter into the systemic circulation.

$\checkmark$ Drugs which require high blood levels to be achieved cannot be used in this delivery system.

$\checkmark$ Drug with lipophilic character is more suitable as compare to drug with hydrophilic character because of their low permeability.

$\checkmark$ Only less quantity of lipophilic drug can be administered through the skin.

\section{MECHANISM OF ACTION OF TRANSDERMAL PATCH}

A typical transdermal patch consists of an adhesive layer which sticks on to the skin, a semi solid to liquid drug is smeared between the layers of drug releasing membranes which are exclusively semipermeable in nature. During application, the entire patch is protected by an outermost transparent backing. When a transdermal patch is placed to the skin, it creates a strong bond between the skin and the semipermeable membrane. Through the percutaneous drug delivery system, a gradual and steady flow of drug is delivered from the patch's drug reservoir to the skin via the drug release membrane via a simple diffusion/osmosis mechanism ${ }^{22}$

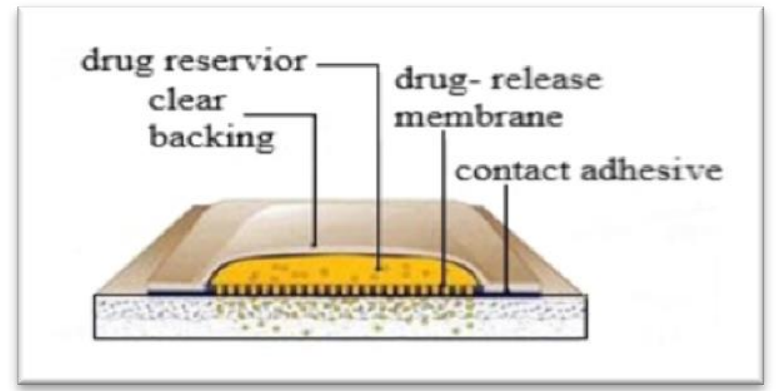

Figure 1: Diagram showing the different layers of transdermal patch with mechanism of action ${ }^{23}$

\section{Basic components of TDDS}

Following are the different types of the parts in the transdermal patch system ${ }^{24}$.

$\checkmark$ Polymer matrix/drug

$\checkmark$ Reservoir Drug

$\checkmark$ Permeation enhancer

$\checkmark$ Adhesive Layer

$\checkmark$ Backing film

$\checkmark$ Release Liner

\section{$\checkmark$ Plasticizer}

It is not necessary that the all 6 components should be present in every transdermal patches. All transdermal patches have the same backing film, medication reservoir, and release liner. The usage of other components is determined by the drug's specifications.

\section{A] Polymer matrix ${ }^{25,26}$}

Polymers are the important component of a transdermal drug delivery system. Multilayered polymeric laminations for controlled release sandwich a drug-polymer matrix or drug reservoir between two polymeric layers: an outer impervious backing layer that prevents drug loss through the backing surface and an inner polymeric layer that serves as an adhesive and/or rate-controlling membrane.

There are different type's polymers which can be used for transdermal delivery systems.

\section{(i). Natural Polymers}

Natural polymers can be utilized to target medication delivery rates at specified intervals. Natural polymers, which are essentially polysaccharides, have no negative side effects and are biocompatible. Natural materials such as mucilages, gums, plant extracts, and resins are frequently employed in both traditional and innovative dosage forms. Natural polymers remain primarily more attractive because of their ready availability, commercial, capable of multitude of chemical modifications, potentially degradable and compatible due to their origin. Polymers are mostly utilized in transdermal drug delivery systems to regulate drug release from the patch/device, but they may also be employed to create a variety of controlled and targeted drug delivery systems. Natural polymers may be utilized to target specified drug delivery rates, and their physico-chemical characteristics, along with the ease of drug availability, give a foundation for using it as a polymer in a transdermal drug delivery system. 


\section{(ii). Synthetic elastomer}

Synthetic elastomer adhesive that are room temperature vulcanizing (RTV) sealants. While these sealants are very useful and interesting class of ingredient in TDDS. RTV sealants are non-tacky and rubbery once fully cross-linked, and are meant to establish a lasting connection between substrates. These compounds have a similar chemistry to polymer caulks used as adherents, and have found usage as synthetic elastomers in TDDS adhering materials.

\section{(iii). Synthetic polymers}

Synthetic polymers are developed for pharmaceutical applications in drug delivery system is reviewed as that synthetic polymers can be used to release a drug in vivo or that can respond to changes in environment to enhance the effectiveness of therapy. The mechanisms by which these polymers are designed to deliver drugs are highlighted, along with the challenges facing polymer synthetic chemists and pharmaceutical dosage form developer in designing new and more active therapeutic vehicles. Two promising synthetic polymers which have been developed for biomedical applications are polyvinyl pryolidone and polyethylene glycol acrylate based hydrogels, these are biodegradable and forms copolymers with natural macromolecules.

Table 1: Examples for three types of polymers used in formulation of TDDS.

\begin{tabular}{|l|l|l|}
\hline Natural Polymers & Synthetic elastomer & Synthetic Polymers \\
\hline $\begin{array}{l}\text { Cellulose derivative, Gelatin, Shellac, } \\
\text { Starch, Waxes, Gums, Natural rubber, } \\
\text { Chitosan etc. }\end{array}$ & $\begin{array}{l}\text { Poly butadiene, Hydrin rubber, } \\
\text { Polyisobutylenes, Silicon rubber, Nitrile, } \\
\text { Acronitryle, Neoprene, Butyl rubber etc }\end{array}$ & $\begin{array}{l}\text { PVA, Polyvinyl chloride, Polyethylene, } \\
\text { PVP, Poly acrylate etc. }\end{array}$ \\
\hline
\end{tabular}

\section{B] Drug Reservoir}

Transdermal drugs release lesser amounts of drug into the blood stream over an extended period of time. These "skin patch" drugs which include nicotine, pain relievers, hormones, and drugs to treat angina and motion sickness. The selection of drug is based on its properties like physiochemical as well as biological properties.

\section{C] Permeation Enhancers}

These are the substances which are reversibly changes the structure of stratum corneum and increase the permeation of drug from skin to blood stream. They are segregated in two types.

\section{(a) Chemical permeation enhancers and (b) Physical permeation enhancers}

a) Chemical permeation enhancers [accelerants, absorption promoters (or) permeation enhancers]: They act by increasing drug permeability by reversible damage to stratum corneum and to increase partition coefficient of drug.

(b) Physical permeation Enhancers:

When drug molecules come into contact with the skin's surface area, they can enter by one of three routes: sweat ducts, hair follicles, and sebaceous glands (known as the shunt or appendageal route), or straight across the stratum corneum. Scientists has been debated over the year about the relative importance of the appendageal or shunt route verses transport across the stratum corneum, and is further difficult by the lack of an appropriate experimental model to permit separation of the three pathways. In vitro experiments have a tendency to involve the use of epidermal membranes or hydrated skin, so that appendages are closed by the swelling associated with hydration. The pre-steady state penetration of polar molecules and flow of large polar molecules or ions that have difficulties diffusing through the entire stratum corneum were thought to be caused via a follicular shunt pathway. However, because the appendages only account for around 0.1 percent of the total permeable surface, their contribution to steady-state drug flow is thought to be negligible. A lot of study has gone into better understanding the barrier characteristics and structure of the stratum corneum. However, it's crucial to keep in mind that corneocytes are elongated, flat, and polygonal (200 - 1500 $\mathrm{nm}$ thick, $34000-46000 \mathrm{~nm}$ in diameter), rather than brickshaped. The intercellular lipid matrix is formed when keratinocytes in the mid to upper stratum granulosum release their lamellar contents into the intercellular space. This extruded material rearranges to produce wide intercellular lipid lamellae 27 in the stratum corneum's early layers, which later associate to form lipid bilayers with hydrocarbon chains aligned and polar head groups dissolved in an aqueous layer. The lipid phase behavior of the stratum corneum varies from that of other biological membranes due to its lipid content. Inside the lipid bilayers, the hydrocarbon chains are arranged into crystalline, lamellar liquid crystal phases, and lamellar gel regions, resulting in various domains. Ionotophoresis, Electrophoresis, Sonophoresis, Microneedling, Magnetophoresis, and Laser Radiation are some of the methods that penetration enhancers use.

\section{D] Adhesive Layer}

The reservoir transdermal system, unlike multi-layer drugin-adhesive systems and single-layer systems, contains a distinct drug layer. The drug reservoir is completely enclosed in a shallow compartment composed of a drugimpermeable metallic plastic laminate with a rate-controlling membrane made of a polymer similar to vinyl acetate on one surface.

The drug layer is separated by special adhesive layer ${ }^{1}$. e.g. polyacrylates, polyisobutylenes and silicone derivatives. 
Table 2: List of Chemical Enhancers Used In TDDS

\begin{tabular}{|l|l|}
\hline \multicolumn{1}{|c|}{ Chemical Enhancers } & \multicolumn{1}{c|}{ Examples } \\
\hline Solvents & Water, Methanol, Ethanol, Propylene Glycol, Di-Methyl Acetamide \\
\hline Terpenes & Menthol, Cardamom Oil, Cinnamon Oil, 18-Cineol, Carvone \\
\hline Pyrolidine & N-Methyl 2- Pyrolidine, Axone \\
\hline Sulfoxides & DMS, Didecyl Sulfoxides \\
\hline Fatty Acids \& Esters & Oleic Acid, Linoleic Acid, Lauric Acid, Capric Acid \\
\hline Surfactants & $\begin{array}{l}\text { Anionic- SLS, Decodecyl Methyl, Sulfomide, Non-Ionic- Pluronic F127, Pluronic F68, Bile Salts- } \\
\text { Sodium Taurocholate, Sodium Deoxy Cholatte }\end{array}$ \\
\hline Amides & Dimethyl Acetamide, Dimethyl Formamide \\
\hline Miscellaneous & Phosphor Lipids, Amino Acid Derivatives, Enzymes, Urea \\
\hline
\end{tabular}

\section{E] Backing Laminate}

Backing films allow the finished transdermal patch easy to remember and easy to visualize, increasing compliance with medication regimens. The patient or a caregiver can write on the film to record the dosing time and dose administered. It can also be printed on both sides with logos, graphics or instructions. e.g. polyester film laminate, polyolefin backing film, ethylene vinyl acetate/polyethylene terephthalate (EVA/PET), polyester/ethylene-vinyl acetate film, ethylenemethacrylic acid copolymer)/ethylene vinyl acetate etc.

\section{F] Release Liner}

During storage of the patches, release liner protects patches from loss of the drug and migration of drug into the adhesive layer and contamination by foreign materials. As a result, it is classified as a component of the principal packing material rather than a component of the dosage form used to release the medication. The release liner is made consisting of a nonocclusive (paper fabric) or occlusive (polyethylene, polyvinylchloride) base layer and a silicon or Teflon release coating layer. Polyester release protective liner, siliconized or fluoropolymer-coated polyester film, siliconized PET release liner covering, paper polyethylene-foil pouch, two overlapped siliconized polyester strips, transparent fluoropolymer-coated polyester film, a peripheral layer of acrylic adhesive, and other materials are used for TDDS release liner.

\section{G] Plasticizer}

Many of the polymers used in pharmaceutical formulations are brittle in nature, which requires plasticizer to reduce the brittleness into the formulation. Plasticizers are added to pharmaceutical polymers aiming to ease the thermal workability, improving the mechanical properties, modifying the drug release from polymeric systems and surface properties of the dosage form. The plasticizers used in pharmaceutical formulations present a) in coating material of solid dosage forms, and b) in transdermal therapeutic systems ${ }^{28}$.

\section{TYPES OF TRANSDERMAL PATCHES}

Transdermal patches or transdermal delivery system is a type of medicated product in which system medicated adhesive patches are available in different sizes and having more than one ingredient. A transdermal patch usually contains more dose of drug inside which is retained on the skin for extended period of time, which get enters into blood circulation through diffusion process.

\section{DRUG PENETRATION THROUGH SKIN:}

Drug penetration through the skin is a key factor in cutaneous responses to xenobiotics, medicines, and other substances. One of the most difficult elements of correctly measuring percutaneous absorption is the size of the compartments. A semisolid dose form, such as a gel, ointment, or cream, is generally administered to a thickness of less than $10 \mathrm{~m}$ when applied topically. The stratum corneum is also around $10 \mathrm{~m}$ thick, whereas the viable epidermis, dermis, and, to a lesser degree, the systemic compartment act as a large sink for ingested toxins, diluting them to levels that are often undetected by all but the most sensitive techniques. As a result, sampling time-dependent changes in a compound's concentration in separate compartments is technically hard ${ }^{29}$.

\section{Drug can penetrate through skin various pathways}

a) Through hair follicular penetration.

b) Through transcorneal penetration.

c) Through intracellular route.

d) Through transcellular route.

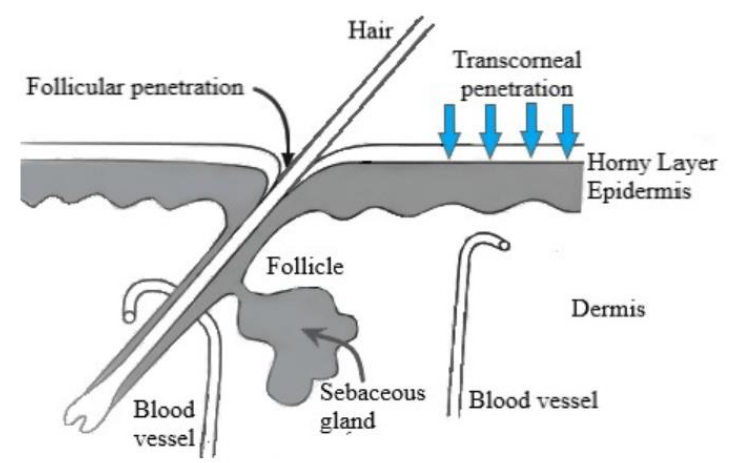

Figure 2: Type of penetration pathways: Penetration happens through hair follicular penetration and transcorneal penetration ${ }^{29}$

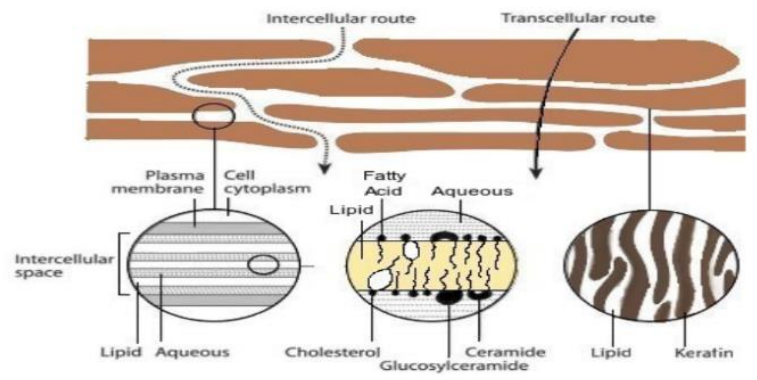

Figure 3: Type of penetration pathways: Penetration happens through intracellular route and transcellular route ${ }^{29}$.

Single-layer drug-in-Adhesive: This system's sticky layer comprises both the medication and the polymer. The sticky layer serves a dual purpose in this sort of patch. The adhesive layer aids in the adhesion of the different layers, as well as the overall system, to the skin, and it is also responsible for medication release. The adhesive layer is covered by a temporary liner and a backing film ${ }^{30}$. e.g. 
Dubey et al

Menostar ${ }^{\circledR}$ (estradiol transdermal system), MINIVELLE ${ }^{\circledR}$ (estradiol transdermal system), CombiPatch ${ }^{\circledR}$ (estradiol/norethindrone acetate transdermal system), DURAGESIC (fentanyl transdermal system), SANCUSO (Granisetron Transdermal System), DAYTRANA ${ }^{\circledR}$ (methylphenidate transdermal system), OXYTROL ${ }^{\circledR}$ (oxybutynin transdermal system), NEUPRO (rotigotine transdermal system), EMSAM $^{\circledR}$ (selegiline transdermal system)

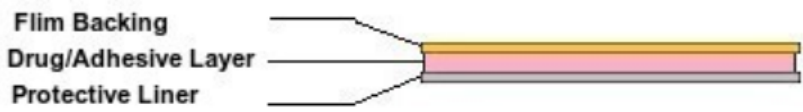

Figure 4: Single-layer drug in adhesive ${ }^{31}$.

Multi-layer drug-in-Adhesive: Adhesive patches with multilayer drugs are similar to single-layer systems in that both adhesive layers are responsible for medication release. Multilayer systems, on the other hand, are distinguished by the addition of a second layer of medicine in adhesive, which is generally separated by a membrane in certain situations. A temporary liner layer and a permanent backing film are also included in this patch ${ }^{32}$. e.g. EXELON ${ }^{\circledR}$ PATCH (rivastigmine transdermal system).

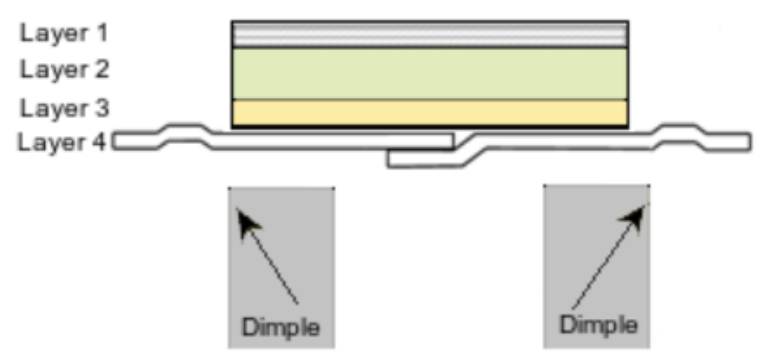

(1) backing layer, (2) drug matrix, (3) adhesive matrix (4) overlapping release liner

Figure 5: Multi-layer drug in adhesive ${ }^{33}$

Reservoir: The Single-layer and Multi-layer Druginadhesive systems are not the same as the Reservoir system. A distinct drug layer exists in the Reservoir transdermal system. The drug layer is in a liquid compartment that has been separated by the adhesive layer and contains drug in a solution or suspension form. The backing layer also supports this patch. By this system zero order release can be achieved. ${ }^{34}$. e.g ANDRODERM $^{\circledR}$ (testosterone transdermal system)

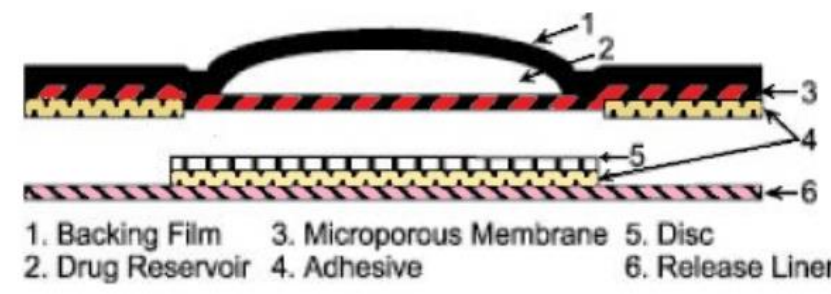

Figure 6: Reservoir 35

Matrix: The drug layer of the Matrix transdermal delivery system is a semisolid matrix containing a drug solution or suspension. This kind of transdermal patch system is partially overlaid by the adhesive layer by surrounding the drug layer ${ }^{36}$. e.g BUTRANS ${ }^{\circledR}$ (buprenorphine) transdermal system
Journal of Drug Delivery \& Therapeutics. 2021; 11(4-S):176-186

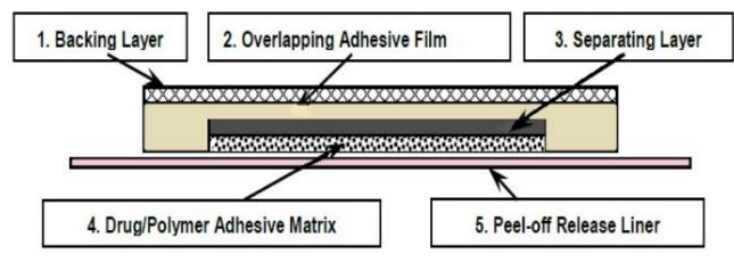

Figure 7: Matrix ${ }^{37}$.

\section{VARIOUS METHODS FOR PREPARATION OF TDDS:}

There are some unique well known methods are available for the preparation of TDDS and those are mentioned below

\section{Circular Teflon Mould Method: -}

In this approach, an organic solvent is utilised to dissolve a solution containing polymers in different ratios. The calculated amount of medicine is dissolved in half of the same organic solvent enhancers in various concentrations are dissolved in the other half of the organic solvent, and then added. As a plasticizer, di-N butyl phthalate is added to the drug polymer solution. The entire mixture is agitated for 12 hours before being placed into a circular Teflon mould. In a laminar flow hood model with an air speed of $0.5 \mathrm{~m} / \mathrm{s}$, the moulds are set on a flat surface and covered with an inverted funnel to regulate solvent vaporisation. For 24 hours, the solvent is allowed to evaporate. The dried films are stored for another $24 \mathrm{~h}$ at $25 \pm 0.5^{\circ} \mathrm{C}$ in a desiccator containing silica gel before evaluation to eliminate aging effects ${ }^{2,38-40}$.

\section{Asymmetric TPX Membrane Method: -}

A prototype patch may be made using a heat sealable polyester film, such as type $1009,3 \mathrm{~m}$, with a $1 \mathrm{~cm}$ diameter concave as the backing membrane. The drug sample is dispensed into the concave membrane, which is then covered with an asymmetric poly (4-methyl-1-pentene) (TPX) membrane and sealed with an adhesive. The dry/wet inversion technique is used to create them. To make a polymer solution, TPX can be dissolved in a combination of solvent (cyclohexane) and nonsolvent additives at $60^{\circ} \mathrm{C}$. The polymer solution is maintained at $40^{\circ} \mathrm{C}$ for 24 hours before being cast on a glass plate using a gardener knife to a predetermined thickness. After that, the casting film is evaporated at $50^{\circ} \mathrm{C}$ for 30 seconds, and the glass plate is immediately submerged in the coagulation bath [at $25^{\circ} \mathrm{C}$ ]. After 10 minutes of immersion in an oven at $50^{\circ} \mathrm{C}$ for 12 hours, the membrane can be withdrawn and air dried in a circulation $2,38-40$.

\section{Mercury Substrate Method: -}

The medication is dissolved in a polymer solution containing a plasticizer in this technique. To achieve a homogeneous dispersion, the aforementioned solution is agitated for 10-15 minutes before being placed over a flat mercury surface and covered with an inverted funnel to prevent solvent evaporation.

\section{"IPM Membranes" Method: -}

Isopropyl myristate (IPM) was the most effective enhancer with the highest permeation flux of $51.763 \mu \mathrm{g} / \mathrm{cm}^{2} / \mathrm{h}$, IPM is a well-tolerated permeation enhancer for topical formulations and is widely used as a nonvolatile solvent in the cosmetic and pharmaceutical industry. IPM is thought to work as a permeation enhancer by disturbing the skin's highly organized lipid structure, therefore changing skin permeability. Because IPM is lipophilic, it has an intrinsic capacity to interact with skin bilayers. Because IPM is intermediately polar, it can partition into the polar phase (proteins) of skin. IPM belongs to the aliphatic esters class of 


\section{Dubey et al}

permeation enhancers, which tend to improve the partition coefficient for skin as well as the diffusivity to skin. The medication is disseminated in a combination of water and propylene glycol containing polymer carbomer 940 and swirled with a magnetic stirrer for 12 hours. With the addition of triethanolamine, the dispersion will be neutralized and viscous. If the drug's solubility in aqueous solution is poor, a buffer $\mathrm{pH} 7.4$ can be employed to make a solution gel. The gel that has been produced will be integrated into the IPM membrane ${ }^{38-40}$.

\section{Aluminium Backed Adhesive Film Method:}

When the loading dose is more than $10 \mathrm{mg}$, the aluminumbacked sticky film technique is appropriate. Because most medicines and adhesives are soluble in chloroform, it is the solvent of choice for the production of the same. The drug particles are dissolved in chloroform, and the adhesive material is added and dissolved in the drug solution. The ends of a custom-made aluminum former are blanked with precisely fitting cork blocks, which are lined with aluminum foil $38-40$.

\section{Preparation of TDDS by Using Proliposomes: -}

The proliposomes are made utilizing a film deposition approach and a carrier method. The proliposomes are made by putting $5 \mathrm{mg}$ of mannitol powder in a $100 \mathrm{ml}$ round bottom flask that is maintained at $60-70^{\circ} \mathrm{C}$ and spun at 80 $90 \mathrm{rpm}$ while drying the mannitol at vacuum for 30 minutes. The temperature of the water bath is changed to $20-30^{\circ} \mathrm{C}$ after drying. The drug is mixed with lecithin and dissolved in an organic solvent combination; a $0.5 \mathrm{ml}$ aliquot of the organic solution is added to the round bottomed flask at $37^{\circ} \mathrm{C}$, and the second aliquot $(0.5 \mathrm{ml})$ is added after the second aliquot $(0.5 \mathrm{ml})$ has dried completely. After the final loading, the flask holding proliposomes is linked in a lyophilizer, and the drug-loaded mannitol powdered proliposomes are desiccated overnight and sieved through 100 mesh. The obtained powder is placed in a glass bottle and kept frozen until it is time to characterize it.

\section{Free Film Method: -}

This approach involves casting a free cellulose acetate film over a mercury surface. Chloroform is used to make a polymer solution ( 2 percent $w / w$ ). Plasticizers should be used at a concentration of $40 \%$ by weight of the polymer. In a glass petridish, $5 \mathrm{~mL}$ of polymer solution was put into a glass ring that was positioned over the mercury surface. Placing an inverted funnel above the petridish controls the pace of solvent evaporation. The film formation is conducted when the solvent has completely evaporated and the mercury surface has been seen. The dried film is separated and kept in a desiccator between wax paper sheets until needed. By varying the amount of the polymer solution, free films of various thicknesses may be created ${ }^{41-46}$.

\section{APPROACHES IN THE DEVELOPMENT OF CONTROLLED DELIVERY TRANSDERMAL THERAPEUTIC SYSTEM}

Many methods have been successfully developed to give rate control over medication release and transdermal penetration. These technologies are as follows:

\section{Adhesive Dispersion System: -}

The system consists of a drug-impermeable backing membrane, a drug reservoir made by directly dispersing the drug substance in an adhesive polymer and then spreading the drug substance added adhesive polymer onto a flat drugimpermeable backing sheet by solvent casting or hot melting to form a thin layer of drug reservoir film. On the upper
Journal of Drug Delivery \& Therapeutics. 2021; 11(4-S):176-186 surface of this with removable release liner which in an ideal situation is removed and the patch is applied to the skin for an adequate period of time, a layer of release controlling adhesive polymer (does not contain medicine) of constant thickness is spread to obtain an adhesive diffusioncontrolled drug delivery system. The development and commercialization of a transdermal therapeutic system for the management of pain in opioid-tolerant individuals, such as the Fentanyl transdermal system for 72-hour therapy, is an example of this sort of system.

\section{Permeation Controlled Membrane System: -}

The drug reservoir system is fully integrated in a compartment moulded between a drug-impermeable backing laminate and a rate-controlling polymeric membrane in this design. The drug molecules are formulated to release across the release controlling membrane by diffusion process through the micro pores. The drug substance is uniformly dispersed in a solid polymeric matrix e.g. polyisobutylene, dispersed in a viscous unleachable liquid medium to obtain a gel-like suspension e.g. silicon fluid, or dissolved in a releasable (e.g. alkyl alcohol) solvent to obtain a gel-like solution in the reservoir compartments. The release regulating membrane can be a microporous or non-porous polymeric membrane with particular drug permeability, such as an ethylene-vinyl acetate copolymer. A small coating of drug suitable adhesive polymer, such as silicone adhesives, can be placed to the top surface of the polymeric membrane to enable intimate contact of the transdermal system with the skin surface. The polymer composition, thickness of the rate-controlling membrane, adhesive, and permeability coefficient may all be tweaked to customise the release rate from this transdermal device.

\section{Matrix Diffusion Controlled System: -}

In this drug reservoir system, the drug reservoirs are prepared by uniformly dispersing drug substance in a lipophilic or hydrophilic polymer matrix or combination of both polymer matrix. The resulting medicated polymer is then moulded into a medicated disc with a predetermined surface area and thickness. Drug substance particles can be dispersed in polymer matrix by homogeneously mixing finely ground drug particles with a liquid polymer or homogeneously blending drug solids with a rubbery polymer at an elevated temperature and/or under vacuum or a highly viscous polymer followed by cross linking of the polymer chains. In a compartment manufactured from a drug-impermeable backing film, the polymer disc containing the drug reservoir is mounted onto an occlusive base plate. The sticky polymer is then applied to the rim and the medicated disc to create a rim strip. The buprenorphine releasing transdermal therapeutic system for the treatment of partial opioid agonist recommended for the management of pain is the greatest example of this matrix kind of transdermal system. The absence of dosage dumping is a benefit of matrix dispersion type transdermal systems since the polymer cannot break.

\section{Micro Reservoir Controlled System: -}

This micro reservoir-controlled system is mixture of matrix dispersion and reservoir type of drug delivery system. In this approach, e.g., silicone elastomers, drug reservoirs are formed by dispersing or spreading the drug in an aqueous solution of liquid polymer to obtain a drug suspension and then dispersing the drug suspension homogeneously in lipophilic polymer by high shear mechanical force to produce thousands of unreachable and microspheres of drug reservoirs. Nitro disc was made possible because to this technology. Depending on the relative magnitude of solubility of the drug in the liquid compartment and the 


\section{Dubey et al}

polymer matrix, drug release from a micro reservoir-type system can follow either a matrix diffusion-control or a partition-control procedure ${ }^{47-49}$.

\section{FUTURE OF TRANSDERMAL DRUG DELIVERY SYSTEM}

Administration of medicines intended for transdermal delivery via liposomes, niosomes, and micro emulsions are among the future elements of transdermal drug delivery systems. The goal of this research is to enhance the delivery of drugs with limited intrinsic solubility in most traditional excipients. Steroids, antifungal, antibacterial, interferon, methotrexate, and local anaesthetics are only a few of the possible medicines for transdermal administration. The market for transdermal patches is expected to rise in the future, with a 25 percent annual growth rate recently discovered. This number is likely to rise in the future as new devices become available and the number of marketed transdermal drugs grows. The popularity of transdermal analgesic administration is anticipated to grow, necessitating additional design improvements. The transdermal patch may be a lesser-known treatment option for chronic and acute pain. We expect the popularity and usefulness of this mode of medication delivery to grow as delivery improves and a larger choice of analgesics becomes available. Therapeutics are being studied in order to improve their safety and efficacy. To increase patient compliance, such as the patch wearer's experience, as well as to offer more accurate medication administration with a longer duration of action. Systemic drug administration through the skin has numerous advantages, including keeping a consistent drug level in blood plasma, fewer adverse effects, improved bioavailability by avoiding hepatic first-pass metabolism, and increased patient compliance with respect to the treatment regimen. Skin is now widely regarded as the safest route for medication delivery since it allows for continuous drug release into the systemic circulation ${ }^{50}$.

\section{PHOSPHODIESTERASE INHIBITORS (PDE-5I) AS A FUTURE DRUG OF CHOICE FOR TDDS}

Phosphodiesterase inhibitor of cGMP specific phosphodiesterase type-5 (PDE-5) in the smooth muscle of the pulmonary vasculature, where $\mathrm{PDE}-5$ is responsible for degradation of cGMP. PDE-5i, therefore by increases cGMP within pulmonary vascular smooth muscle cells would resulting in relaxation. This can lead to vasodilation of the pulmonary vascular bed and, to a lesser degree, vasodilatation in the systemic circulation to the patients with PAH. Hence Phosphodiesterase inhibitor (PDE-5i) as a drug of choice for transdermal delivery system ${ }^{51}$.

\section{There are two reasons given by different scientist}

1. Phosphodiesterase Inhibitors (PDE-5i) ${ }^{74}$ i.e sildenafil, tadalafil, vardenafil and avanafil , are used for the treatment of male penile erectile dysfunction through selective inhibition of phosphodiesterase type-5 (PDE-5) to decrease the metabolism of cGMP, which induces smooth muscle relaxation in the corpus cavernosum with onset around 30 $45 \mathrm{~min}^{52}$. However, blood pressure is transiently reduced by oral administration of $100 \mathrm{mg}$ of sildenafil followed by the possible adverse effect of color blindness (blue / green) discrimination, headaches, flushing, and nasal congestion ${ }^{53}$. In the meantime, repeated doses are required to sustain plasma levels because of its short duration of action $(t 1 / 2$ $=1 \mathrm{~h}$ ) with high liver metabolism ${ }^{52}$. Thus, topical delivery through a local tissue area could be considered as alternative administration, instead of oral route to avoid above mentioned adverse effects, to shorter onset time, and
Journal of Drug Delivery \& Therapeutics. 2021; 11(4-S):176-186

to sustain effect for longer periods. Furthermore, transdermal delivery system using sildenafil as a therapeutic agent can offer several advantages than the conventional dosage forms of sildenafil. However, transdermal permeation of compounds in the local skin, in general, is slow due to low permeability for reasons including the physicochemical properties of the compound, low partition ability, and the tissue barrier from the stratum corneum creating a low diffusion coefficient54. In addition, the structure of sildenafil has basic functional groups whose $\mathrm{pKa}$ value is 6.555 and under different $\mathrm{pH}$ environments, sildenafil will have different levels of ionization following the influence of the partition coefficient as well as its permeation ability in the skin.

2. Recently, Phosphodiesterase Inhibitors (PDE-5i) have been used for the treatment of pulmonary arterial hypertension and the enhancement of uteroplacental perfusion in case of fetal growth retardation 56,57 . Sildenafil, on the other hand, has a low oral bioavailability (40\%) due to its low water solubility $(3.5 \mathrm{mg} / \mathrm{mL})$ and significant presystemic metabolism, as well as absorption obstruction by food, particularly fat-rich meals ${ }^{58,59-63}$. Researchers have been drawn to the study of SLD delivery via various methods of administration as a means of overcoming this issue. Orally disintegrating and orally dissolving films, intranasal microemulsions, and transdermal nanostructure lipid carriers and solid lipid nanoparticles are only a few of the SLD formulations that have been created to bypass the peroral route ${ }^{64-68}$. Due to avoiding the presystemic and first pass metabolism of medicines, transdermal administration of pharmaceuticals through the skin provides a leading option to the oral route, extending their impact and minimizing inter- and intrasubject variability69. Because of the stratum corneum's inherent barrier function, transdermal medication administration has a major challenge: poor permeation across the skin (SC) ${ }^{70}$. Multiple techniques have been used in recent years to promote medication penetration through the skin, including chemical approaches, such as the use of drug permeation enhancers, as well as physical ones such as sonophoresis and iontophoresis 71,72 . The use of lipid vesicular formulations for improving transdermal medication delivery has also gotten a lot of interest ${ }^{73}$.

\section{CONCLUSION}

Transdermal drug delivery is a safest, less pain, comfortable, and potentially active route to deliver regular doses for many medications. Wide range of drugs can be delivered for improved drug uptake, minimal complications and side effects low cost and easy to use. Transdermal route of drug delivery system has been one of the advanced technique modes of drug delivery with good efficacy and safety. There has been a lot of improvement in the process of transdermal route of drug delivery since its beginning years of 1981 to the current advancements till 2019. TDDS review articles give useful information on the transdermal drug delivery systems method of preparing transdermal patches and numerous medicines that might be packaged as TDDS as a quick reference for researchers working on TDDS development. Moreover transdermal system for PAH treatment to the children and adult are not established so far. Many new researches are going on in the current era to integrate newer drugs via this system. Transdermal dosage forms may provide healthcare providers an additional opportunity to offer more therapeutic modes to their patients to ensure their care. Considering the fact of patient comfortability and to avoid solid and semisolid oral or intravenous injection, transdermal patch with PDE 
Dubey et al

inhibitors would be a best choice of dosage form for the treatment of Pulmonary arterial hypertension.

\section{CONFLICT OF INTEREST:}

The authors have no conflicts of interest.

\section{ACKNOWLEDGEMENT:}

\section{Declared none}

\section{REFERENCES}

1. Dipen Patel, Sunita A. Chaudhary, Bhavesh Parmar, Nikunj Bhura, Transdermal Drug Delivery System: A Review, The Pharma Innovation. 2012; 1(4):66-75.

2. Nidhi S. A Brief Review on Transdermal Patches. Organic \& Medicinal Chem IJ. 2018; 7(2):55570.

3. Hao, Y.; Li, W.; Zhou, X. L.; Yang, F.; Qian, Z. Y. Microneedles-based transdermal drug delivery systems: A review. J. Biomed. Nanotechnol.2017; 13:1581-1597. https://doi.org/10.1166/jbn.2017.2474

4. Barry BW. Mode of action of penetration enhancers in human skin. J Control Release 1987; 6: 85-97. DOI: https://doi.org/10.1016/0168-3659(87)90066-6

5. Marwah H, Garg T, Goyal AK, Rath G. Permeation enhancer strategies in transdermal drug delivery. Drug Deliv. 2016; 23(2):564-578.

https://doi.org/10.3109/10717544.2014.935532

6. Meng S, Zhang C, Shi W, Zhang XW, Liu DH, Wang P, Li JX, Jin Y. Preparation of osthole-loaded nano-vesicles for skin delivery: Characterization, in vitro skin permeation and preliminary in vivo pharmacokinetic studies. Eur J Pharm Sci. 2016 Sep 20; 92:49-54. DOI: https://doi.org/10.1016/j.ejps.2016.04.033

7. Arti Kesarwani, Ajit Kumar Yadav, Sunil Singh, Hemendra Gautam, Haribansh N Singh. A review-Theoretical aspects of Transdermal Drug Delivery System. Bulletin of Pharmaceutical Research. 2013; 3(2):78-89.

8. Venkatraman S, Gale R. Skin adhesives and skin adhesion 1. Transdermal drug delivery systems. Biomaterials 1998; 19(13):1119-36. https://doi.org/10.1016/S01429612(98)00020-9

9. Wu J, Nyborg W, editors. Emerging Therapeutic Ultrasound. London: Imperial College Press; 2006. https://doi.org/10.1142/6047

10. Z. A. M. Yasin, F. Ibrahim, N. N. Rashid, M. F. M. Razif, and R. Yusof. The importance of some plant extracts as skin anti-aging resources: a review. Current Pharmaceutical Biotechnology. 2017; 18 (11):864-876 https://doi.org/10.2174/1389201019666171219105920

11. Ventrelli, L.; Strambini, L.M.; Barillaro, G. Microneedles for transdermal biosensing: current picture and future direction. Adv. Healthcare Mater. 2015; 4:2606-2640. https://doi.org/10.1002/adhm.201500450

12. Zhao C, Quan P, Liu C, Li Q, Fang L. Effect of isopropyl myristate on the viscoelasticity and drug release of a drug-in-adhesive transdermal patch containing blonanserin. Acta Pharm Sin B. 2016; 6:623-628. https://doi.org/10.1016/j.apsb.2016.05.012

13. Kodiweera C, Yang Y, Bunge AL. Characterization of water selfdiffusion in human stratum corneum, J Pharm Sci 2018; 107:1131-1142. https://doi.org/10.1016/j.xphs.2017.12.011

14. Ma M, Di HJ, Zhang H, Yao JH, Dong J, Yan GJ, Qiao HZ, Chen J. Development of phospholipid vesicle-based permeation assay models capable of evaluating percutaneous penetration enhancing effect. Drug Dev Ind Pharm. 2017 Dec; 43(12):2055 2063. DOI: https://doi.org/10.1080/03639045.2017.1371730

15. Cevc G, Vierl U. Nanotechnology and the transdermal route: A state of the art review and critical appraisal. J Control Release. 2010; 141(3):277-299.

https://doi.org/10.1016/j.jconrel.2009.10.016
Journal of Drug Delivery \& Therapeutics. 2021; 11(4-S):176-186

16. Armengot-Carbo M, Hernández-Martín Á, Torrelo A. The role of filaggrin in the skin barrier and disease development. Actas Dermosifiliogr. 2015; 106(2):86-95. https://doi.org/10.1016/j.ad.2013.10.019

17. Illel B, Schaefer H, Wepierre J, Doucet O. Follicles Play an important role in percutaneous absorption. J Pharm Sci 1991; 80:424-427. https://doi.org/10.1002/jps.2600800505

18. Erdő F, Hashimoto N, Karvaly G, Nakamichi N, Kato Y. Critical evaluation and methodological positioning of the transdermal microdialysis technique. A review. J Control Release. 2016; 233:147-161. https://doi.org/10.1016/j.jconrel.2016.05.035

19. Rohini Rana1, Kamal Saroha, Uditi Handa, Ajay Kumar and Sanju Nanda. Transdermal Patches as a tool for permeation of drug through skin. Journal of Chemical and Pharmaceutical Research. 2016; 8(5):471-481.

20. Leya mathews, Anitha roy. Management of pain using transdermal patches - a review. Asian J Pharm Clin Res. 2016; 9(6):32-35. https://doi.org/10.22159/ajpcr.2016.v9i6.13775

21. Trommer H, Neubert RH. Overcoming the stratum corneum: the modulation of skin penetration. A review. Skin Pharmacology and Physiology. 2006; 19(2):106-121 https://doi.org/10.1159/000091978

22. Neelkanth Kote, Poornima B. Transdermal Patch: A Comprehensive Overview of Newer Drug Delivery System in Modern Medical Science. Journal of Medical Science and clinical research 2007; 5(1):15625-15629. https://doi.org/10.18535/jmscr/v5i1.71

23. Verma PRP and Iyer SS. Transdermal delivery of propranolol using mixed grades of eudragit: Design and in vitro and in vivo evaluation. Drug Dev Ind Pharm. 2000; 26:471-476. https://doi.org/10.1081/DDC-100101257

24. ANDRODERM® (testosterone transdermal system), Initial U.S. Available at: https://www.accessdata.fda.gov/drugsatfda_docs/label/2016/ 020489s034lbl.pdf 29 June, 2020.

25. Boretos JW, Detmer DE, Donachy JH. Segmented polyurethane: a polyether polymer, II. Two years experience. J Biomed Mater Res. 1971; 5(4):373-387 https://doi.org/10.1002/jbm.820050408

26. Barry BW. Action of skin penetration enhancers-the Lipid Protein Partitioning theory. Int J Cosmet Sci. 1988 Dec; 10(6):281-93. DOI: https://doi.org/10.1111/j.14672494.1988.tb00028.x

27. Rangari N.T., Kalyankar T.M., Puranik P.K., Chaudhar S.R. Permeation Studies Of Pioglitazone Hcl From Ficuscarica Fruit Mucilage Matrix Transdermal Patches, International Journal of Pharmaceutical Sciences and Research. 2012; 3(10):3927-3931

28. Sevgi Güngör, M. Sedef Erdal and Yıldız Özsoy. Plasticizers in Transdermal Drug Delivery Systems, Recent Advances in Plasticizers, Dr. Mohammad Luqman (Ed.), 2012. ISBN: 978953-51-0363-9, InTech, Available at: https://cdn.intechopen.com/pdfs/32871/InTechPlasticizers_in_transdermal_drug_delivery_systems.pdf 30 June, 2020.

29. Hans Schaefer, Thomas E. Redelmeier. Skin Penetration. Chepter 11; 167-176, available at: http://eknygos.lsmuni.lt/springer/99/167-178.pdf 30 June, 2020. https://doi.org/10.1007/3-540-31301-X_11

30. Ghafourian T, Zandasrar P, Hamishekar H, Nokhodchi A. The Effect of Penetration Enhancers on Drug Delivery through Skin. J Control Release. 2004; 99:113-25. https://doi.org/10.1016/j.jconrel.2004.06.010

31. Menostar $®$ (estradiol transdermal system) Initial U.S. Available at: https://www.accessdata.fda.gov/drugsatfda_docs/label/017/0 20375s035lbl.pdf 26 June, 2020.

32. Montia D, Saettone Mf, Giannaccini B, Angeli Dg. Enhancement of Transdermal Penetration of Dapiprazole Through Hairless 
Mouse Skin. J Control Release.1995; 33:71-77.

https://doi.org/10.1016/0168-3659(94)00079-A

33. EXELON® PATCH (rivastigmine transdermal system) Initial U.S. Available at:

https://www.accessdata.fda.gov/drugsatfda_docs/label/2018/ 022083s026lbl.pdf 27 June, 2020.

34. Bharadwaj S, Gupta Gd, Sharma Vk. Topical Gel: A Novel Approach for Drug Delivery. Journal of Chemical, Biological and Physical Sciences 2012; 2(2):856-867.

35. ANDRODERM ${ }^{\circledR}$ (testosterone transdermal system), Initial U.S. Available at:

https://www.accessdata.fda.gov/drugsatfda_docs/label/2016/ 020489s034lbl.pdf 28 June, 2020.

36. Sharma N, Parashar B, Sharma S, Mahajan U. Blooming Pharma Industry With Transdermal Drug Delivery System. Indo Global J Pharm. Sci. 2012; 2(3):262-278.

37. BUTRANS® (buprenorphine) transdermal system CIII Initial U.S. Available at:

https://www.accessdata.fda.gov/drugsatfda_docs/label/2019/ 021306s035lbl.pdf 28 June, 2020

38. Tanwar H. and Sachdeva R. Transdermal drug delivery system: A review. Int. J. Pharm. Sci. Res. 2016; 7:2274- 2290.

39. Kadam AS, Ratnaparkhi MP, Chaudhary SP. Transdermal drug delivery: an overview. Int J Res Dev Pharm Life Sci. 2014; 3:1042-1053.

40. A. Kharia, R. Gilhotra and A. K. Singhai. Overview of Transdermal Medicated Patches with its research updates in preceding years. Journal of Drug Delivery \& Therapeutics. 2019; 9(3-s):10941102.

41. Berner B, John Va. Pharmacokinetic Characterization of Transdermal Delivery System. J. Clin. Pharmaco. 1994; 26(2):121-134. https://doi.org/10.2165/00003088199426020-00005

42. Arunachalam A, Karthikeyan M, Kumar Vd, Prathap M, Sethuraman S, Ashutoshkumar S, Manidipa S. Transdermal Drug Delivery System: A Review. Current Pharma Res. 2010; 1(1):7081. https://doi.org/10.33786/JCPR.2010.v01i01.015

43. Kumar Sr, Jain A, Nayak S. Development and Evaluation of Transdermal Patches of Colchicine. Der Pharmacia Lettre. 2012; 4(1):330-343.

44. Patel, D., Sunita, A., Parmar B., Bhura N., Transdermal Drug Delivery System: A Review. The Pharma Innovation. 2012; 1(4):66-75. https://doi.org/10.31638/IJPRS.V1.I1.00018

45. Sharma N., Agarwal G., Rana A.C., Ali Bha Tz.,Kumar D. A Review: Transdermal Drug Delivery System: A Tool for Novel Drug Delivery System. International Journal of Drug Development \& Research. 2011; 3(3):70-84

46. Das, U. S., Pande K.H., an Overview of Diabetes Mellitus, World Journal of Pharmacy and Pharmaceutical Sciences. 2013; 2(1):161-178.

47. Kim Mk, Zhao H, Lee Ch, Kim D.D. Formulation Of A ReservoirType Testosterone Transdermal Delivery System .Int J Pharm. 2011; 219(1-2):51-59. https://doi.org/10.1016/S03785173(01)00631-7

48. M. Aqil and Asgarali, Monolithic Matrix Type Transdermal Drug Delivery Systems of Pinacidil Monohydrate: In Vitro Characterisation. European Journal of Pharmaceutics and Biopharmaceutics. 2001: 54:161-164. https://doi.org/10.1016/S0939-6411(02)00059-0

49. Dhiman S, Transdermal Patches: A Recent Approch To New Drug Delivery System. International Journal of Pharmacy and Pharm 2011; 3(5).

50. Boolell M, Gepi-Attee S, Gingell JC, Allen MJ. Sildenafil, a novel effective oral therapy for male erectile dysfunction. Br J Urol. 1996; 78(2):257-261. https://doi.org/10.1046/j.1464410X.1996.10220.x
Journal of Drug Delivery \& Therapeutics. 2021; 11(4-S):176-186

51. REVATIO (sildenafil) tablets,for oral use; REVATIO (sildenafil) for oral suspension; REVATIO (sildenafil) injection, for intravenous use; Initial U.S. Available at: https://www.accessdata.fda.gov/drugsatfda_docs/label/2019/ 022473s012,203109s012lbl.pdf 27 June, 2020.

52. Moreira SG Jr, Brannigan RE, Spitz A, Orejuela FJ, Lipshultz LI, Kim ED. Side-effect profile of sildenafil citrate (Viagra) in clinical practice. Urology. 2000; 56(3):474-476. https://doi.org/10.1016/S0090-4295(00)00649-X

53. Mitragotri S. Synergistic effect of enhancers for transdermal drug delivery. Pharm Res. 2000; 17(11):1354-1359. https://doi.org/10.1023/A:1007522114438

54. Langtry HD, Markham A. Sildenafil: A review of its use in erectile dysfunction. Drugs 1999; 57:967-989. https://doi.org/10.2165/00003495-199957060-00015

55. Milger K, Felix JF, Voswinckel R, et al. Sildenafil versus nitric oxide for acute vasodilator testing in pulmonary arterial hypertension. Pulm Circ. 2015; 5(2):305-312. https://doi.org/10.1086/680355

56. Dastjerdi MV, Hosseini S, Bayani L. Sildenafil citrate and uteropla 7 cental perfusion in fetal growth restriction. J Res Med Sci. 2012; 17:632-636.

57. Osman MA, El Maghraby GM, Hedaya MA. Intestinal absorption and presystemic disposition of sildenafil citrate in the rabbit: evidence for site-dependent absorptive clearance. Biopharm Drug Dispos. 2006; 27:93-102. https://doi.org/10.1002/bdd.487

58. Rubin LJ, Badesch DB, Fleming TR, et al. Long-term treatment with sildenafil citrate in pulmonary arterial hypertension: the SUPER-2 study. Chest 2011; 140:1274-1283. https://doi.org/10.1378/chest.10-0969

59. Zinner N. Do food and dose timing affect the efficacy of sildenafil? A randomized placebo-controlled study. J Sex Med. 2007 Jan; 4(1):137-144. DOI: htts//doi.org/10.1111/j.17436109.2006.00400.x https://doi.org/10.1111/j.17436109.2006.00400.x

60. Hosny KM, Aljaeid BM. Sildenafil citrate as oral solid lipid nanopar $\neg$ ticles: a novel formula with higher bioavailability and sustained action for treatment of erectile dysfunction. Expert Opin Drug Deliv. 2014; 11:1015-1022. https://doi.org/10.1517/17425247.2014.912212

61. Sawatdee S, Phetmung H, Srichana T. Sildenafil citrate monohydrate-cyclodextrin nanosuspension complexes for use in metered-dose inhalers. Int J Pharm. 2013; 455:248-258. https://doi.org/10.1016/j.ijpharm.2013.07.023

62. Hosny KM, Mosly HA, Hassan AH. Soy polysaccharide as a novel superdisintegrant in sildenafil citrate sublingual tablets: preparation, characterization, and in vivo evaluation. Drug Des Devel Ther. 2015; 9:465-472. https://doi.org/10.2147/DDDT.S76314

63. Kim EY, Lee SY, Jeon JY, Im YJ, Kim Y, Kim HI, Shin KS, Park SJ, Seo YH, Lee JY, Park JK, Chae SW, Kim MG. Pharmacokinetics of a new orally soluble film formulation of sildenafil administered without water. Int J Clin Pharmacol Ther. 2014 Jun; 52(6):43745. DOI: https://doi.org/10.5414/CP201995

64. Damle B, Duczynski G, Jeffers BW, Crownover P, Coupe A, LaBadie RR. Pharmacokinetics of a novel orodispersible tablet of sildenafil in healthy subjects. Clin Ther. 2014; 36:236-244. https://doi.org/10.1016/j.clinthera.2013.12.010

65. Zayed R, Kamel A, Shukr M, El-Shamy A. An in vitro and in vivo comparative study of directly compressed solid dispersions and freeze dried sildenafil citrate sublingual tablets for management of pulmonary arterial hypertension. Acta Pharm. 2012; 62:411432. https://doi.org/10.2478/v10007-012-0027-9

66. Elshafeey AH, Bendas ER, Mohamed OH. Intranasal microemulsion of sildenafil citrate: in vitro evaluation and in vivo pharmacokinetic study in rabbits. AAPS Pharm Sci Tech. 2009; 10:361-367. https://doi.org/10.1208/s12249-009-92136 
Dubey et al

67. Elnaggar YS, El-Massik MA, Abdallah OY. Fabrication, appraisal, and transdermal permeation of sildenafil citrate loaded nanostructured lipid carriers versus solid lipid nanoparticles. Int J Nanomedicine. 2011; 6:3195-3205. https://doi.org/10.2147/IJN.S25825

68. Barry B. Transdermal drug delivery. In: Aulton ME, editor. Phar $\neg$ maceutics: The Science of Dosage Form Design. London: Churchill Livingstone; 2002:499-528.

69. Paudel KS, Milewski M, Swadley CL, Brogden NK, Ghosh P, Stinchcomb AL. Challenges and opportunities in dermal/transdermal delivery. Ther Deliv. 2010; 1:109-131. https://doi.org/10.4155/tde.10.16

70. Mutalik S, Parekh HS, Davies NM, Udupa N. A combined approach of chemical enhancers and sonophoresis for the transdermal delivery of tizanidine hydrochloride. Drug Deliv. 2009; 16:82-91. https://doi.org/10.1080/10717540802605053
Journal of Drug Delivery \& Therapeutics. 2021; 11(4-S):176-186

71. Schoellhammer CM, Blankschtein D, Langer R. Skin permeabilization for transdermal drug delivery: recent advances and future prospects. Expert Opin Drug Deliv. 2014; 11:393-407. https://doi.org/10.1517/17425247.2014.875528

72. Shaimaa M Badr-Eldin Osamaa AAAhmed. Optimized nanotransfersomal films for enhanced sildenafil citrate transdermal delivery: ex vivo and in vivo evaluation. Drug Design, Development and Therapy. 2016; 10:1323-1333. https://doi.org/10.2147/DDDT.S103122

73. Soloman E, Karthik A, Drug Delivery System - Transdermal, International Journal of Pure and Applied Mathematics. 2018, 119 (12):11887-11895.

74. Dubey R, Pothuvan U, Bhamare P, Singh A, Upmanyu N. A Review on Drug of Pediatric Pulmonary Arterial Hypertension (PAH), their Chemistry and Pharmaceutical Dosage Forms. Journal of Drug Delivery \& Therapeutics. 2020; 10(2-s):156-170. https://doi.org/10.22270/jddt.v10i2-s.3947 